\title{
Retorno econômico do aproveitamento de resíduos gerados na produção de frutas no semiárido cearense
}

Economic return of the residues generated in fruit production in the semi-arid region of Ceará

\author{
Ariana Carvalho Pinto ${ }^{1}$; Fernanda Aparecida Castro Pereira ${ }^{2 *}$
}

\begin{abstract}
${ }^{1}$ Agrícola Famosa LTDA. Engenheira Agrônoma. Fazenda Famosa, Sítio Gravier s/n, Zona Rural - 62810-000 - Icapuí, CE - Brasil
${ }^{2}$ Escola Superior de Agricultura Luiz de Queiroz da Universidade de São Paulo - Doutora em Genética e Melhoramento de Plantas e Especialista em Agronegócio, Av. Pádua Dias, 11 - 13418-900 - Piracicaba, SP - Brasil<fernandacpereira01@gmail.com>
\end{abstract}

\section{Resumo}

O reaproveitamento dos resíduos plásticos pode se tornar uma atividade agrícola economicamente viável, principalmente na fruticultura localizada no semiárido cearense. Os materiais nos quais eram tidos como lixo nas áreas agrícolas podem ser uma fonte alternativa de geração de capital e empregos para a região, utilizando-se técnicas de reaproveitamento e reciclagem desses materiais. O objetivo deste trabalho foi avaliar a importância e a viabilidade econômica da reutilização dos materiais plásticos empregados diretamente na fruticultura. Os dados foram coletados de uma empresa agrícola de fruticultura e de uma empresa de reciclagem no município de Icapuí, no estado do Ceará. Foram calculados indicadores de viabilidade financeira como Valor Presente Líquido [VPL], Taxa Interna de Retorno [TIR], Payback simples [PB], Payback descontado [PBD]. Os resultados mostraram que a utilização de técnicas e equipamentos de reciclagem em empresas é um caminho que gera muitas oportunidades de reutilização de materiais antes descartados, bem como a viabilidade econômica, confirmada pelos valores obtidos de VPL (R \$ 529.777,58) e TIR de 31\%. Conforme o cálculo do Payback descontado, serão necessários dois anos e quatro meses para recuperar o investimento dos fluxos líquidos de caixa descontados. Conclui-se que a atividade é sustentável e rentável, trazendo benefícios e lucro ao fruticultor, além de disponibilizar empregos diretos e indiretos.

Palavras-chave: fruticultura, mulching, viabilidade econômica

\begin{abstract}
The reuse of plastic can become an economically viable agricultural activity, mainly in the fruticulture located in the semi-arid region of Ceará. The materials in which they were treated as garbage in the agricultural areas can be an alternative source of capital generation and jobs for the region, using reuse and recycling techniques. The objective of this work was to evaluate the importance and economic viability of the reuse of plastic materials used directly in the fruticulture. The data were collected from an agricultural company of fruit production and recycling at Icapuí, in the State of Ceará. Economic indicators of viability were calculated as Net Present Value [NPV], Internal Rate of Return [IRR], Simple Payback [PB], Discounted Payback [DPB]. The results showed that the use of recycling techniques and equipment in companies is a path that generates many opportunities for reuse of materials previously discarded, as well as economically viable, confirmed by the values obtained from NPV (R $\$ 529.777,58$ ) and IRR of $31 \%$. According to the discounted Payback calculation, it will take two years and four months to recover the investment from discounted cash flows. The conclusion is that the activity is sustainable and economically profitable, bringing benefits and profit to the producers, besides providing direct and indirect jobs.
\end{abstract}

Keywords: fruticulture, mulching, economic feasibility

\section{Introdução}

Com as novas tecnologias que permitiram maior eficiência na produção e acondicionamento de alimentos, surgiram novas fibras e materiais plástico derivados de fontes naturais ou tratamentos químicos a fim de auxiliar na produção agrícola, de forma a expandir o tempo de duração do alimento na prateleira ou mesmo facilitar o manejo e evitar o desperdício de recursos (Neves, 1994).

O plástico tem diversas aplicações, uma delas é na agricultura, para utilização como cobertura de solo e sistema de irrigação (mulching), desde meados da década de 80, sua utilização tem aumentado em importância, principalmente no sul do Brasil. São inúmeras vantagens, dentre elas: evitar desperdício de água, proteger implementos agrícolas (máquinas), cobrir frutas para evitar ataques de insetos e fitopatógenos, embalar frutas para uma melhor apresentação ao cliente, embalar defensivos e adubos, dentre outros (Thompson et al., 2009). Mais especificamente na fruticultura, pode ser utilizado na cobertura de pequenas áreas ou camalhões. Com a utilização de mulching, é possível observar precocidade de produção e aumento da produtividade, 
menor compactação do solo e redução de plantas daninhas (Lamont Jr., 2005).

O material plástico adequado para cada função possui grande capacidade de resistência a contatos físicos, alteração de temperaturas, contato com água e agentes químicos. Também possui baixa predisposição para decomposição por condições climáticas e atuação de microrganismos. Caso ocorra a degradação do material plástico de forma muito acentuada e contínua por má utilização ou escolha equivocada do polímero, pode ocorrer acumulo de resíduos químicos derivado dos plásticos no local no qual foi implantado, implicando em problemas e desequilíbrios ambientais consideráveis, devido a sua composição molecular densa e resistente a intempéries (Kyrikou e Briassoulis, 2007).

Em face da necessidade da utilização de polímeros plásticos na agricultura, mais especificamente na fruticultura, é importante considerar a forma de aplicação deste material, não obstante, o manejo adequado e a finalidade propícia fazem com que o agricultor necessite planejar o descarte (Espí et al., 2016). Uma das utilizações mais congruentes é o seu reuso, pois a composição química dos plásticos permitem que sejam reutilizados através de processos de reciclagem (Pinto et al., 2012).

Canevarolo Jr. (2004) e Akcelrud (2007) afirmaram que polímeros são macromoléculas caracterizadas por seu tamanho, sua estrutura química e interações intra e intermoleculares. Os polímeros são produtos que por sua composição podem demorar séculos para se degradar e como ocupam boa parte do volume dos aterros sanitários, causam problemas sérios no processo de compostagem e de estabilização biológica. Esta situação se agrava quando, de forma inadequada ou criminosa, estes produtos são depositados em lixões, beira de rios e lagos, encostas e outros locais, causando um impacto ambiental de enormes proporções (Piva et al., 1999).

$\mathrm{O}$ reuso deste material além de minimizar os impactos ambientais derivados da prática da fruticultura, pode ser utilizado como fonte de renda secundária, pois a reciclagem dos plásticos é viável através de processos e tratamentos físico-químicos. Esta prática é bem aceita pelos consumidores, visto que os plásticos reciclados possuem qualidade, durabilidade e não agridem ao

(A)

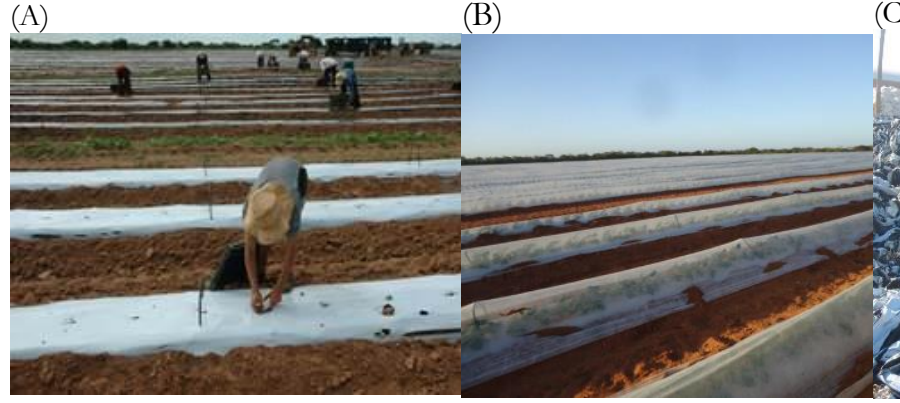

ecossistema. A reciclagem não só possibilita o aumento da vida útil dos materiais, gerando novos negócios empresariais, como também contribui para a proteção ambiental (Rujnić-Sokele e Pilipović, 2017).

Desta forma, a retirada de toneladas de polímeros plásticos do campo de cultivos de frutíferas comerciais, é de grande valia, podendo ser reutilizado de forma intensiva e extensiva em outras áreas das quais se beneficiam por seu baixo preço e grande durabilidade. É considerada uma atividade sustentável pois evita a retirada contínua de matéria prima do meio ambiente, realizadas pelas indústrias, ou o gasto desnecessário para a fabricação do mesmo material, já que o campo pode fornecê-lo para que seja reempregado nas mesmas ou até mesmo outras atividades (Gu et al., 2017).

Este trabalho objetivou avaliar a importância e a viabilidade econômica do processo de reciclagem dos materiais plásticos empregados diretamente na fruticultura.

\section{Material e Métodos}

\section{Fonte de dados}

A empresa agrícola de fruticultura onde foi realizada a pesquisa, localiza-se no município de Icapuí, no estado do Ceará, na fronteira com o estado do Rio Grande do Norte. Paralelamente às atividades produtivas do setor de fruticultura, a empresa apresenta outras atividades agropecuárias, gerando fonte alternativa de renda o que a torna mais competitiva. Dois materiais são destinados à reciclagem, o polietileno (mulching), utilizado na cobertura do solo (Figura 1A) e o polipropileno (Manta/TNT), utilizado sobre os camalhões servindo de barreira física contra entrada de insetos e consequente transmissão de doenças (Figura 1B).

O processo de reciclagem é basicamente igual para os dois. Cada um dos polímeros possui um local de armazenagem correspondente e são processados separadamente para que não haja contaminação entre os diferentes compostos químicos, podendo haver perda de qualidade e durabilidade do material final reciclado (Figuras 1C e 1D).

Figura 1. (A) Uso do mulching em plantios de melão e melancia, safra 2014-2015, Icapuí, Ceará; (B) Manta aplicada sobre os camalhões, Safra 2014-2015. Icapuí, Ceará; (C) Mulching após o cultivo na área de seleção, pronto para serem reciclados; e (D) Manta após o cultivo na área de seleção, pronto para serem reciclados. Safra 2014-2015. Icapuí-Ceará 
O plantio da safra 2014-2015 deu-se em uma área de 8.000 hectares, com plantios de melão e melancia, onde todas as linhas foram cobertas por mulching. Um hectare plantado destas culturas corresponde a cinco mil metros lineares, o mulching empregado na empresa possui 1,1 $\mathrm{m}$ de largura, dessa forma, no total, foram utilizados 44 milhões de $\mathrm{m}^{2}$ de material. E foram utilizados em torno de 24 milhões de $\mathrm{m}^{2}$ de polipropileno (Manta/TNT).

\section{Análise econômica}

Foi realizado um levantamento do custo de produção mensal, capacidade de produção da usina e renda líquida gerada para o material plástico mais utilizado na empresa de fruticultura. Para avaliação da receita criou-se um demonstrativo de despesas e lucro líquido para o processo durante um mês de trabalho. Durante esse mês somente a manta foi processada, embora o processo e os valores são considerados similares para os dois tipos de plásticos.

O fluxo de caixa resume as entradas e as saídas efetivas de dinheiro ao longo do horizonte de planejamento do projeto, permitindo, dessa maneira, conhecer a sua rentabilidade e viabilidade econômica (Samanez, 2002).

Os ingressos foram considerados os valores recebidos pela venda do plástico, as saídas foram representadas pelo investimento inicial e valores investidos mensalmente para a reciclagem.

O objetivo do VPL foi encontrar projetos ou alternativas de investimento que gerem mais receitas que custos, viabilizando o processo (Samanez, 2009). O cálculo foi baseado na eq. (1), ou seja, na subtração dos valores do fluxo de caixa (valor obtido durante um mês de trabalho com capacidade máxima de produção da usina), menos o investimento do capital total do projeto.

$$
\mathrm{VPL}=\left[\frac{\mathrm{FC}_{1}}{(1+\mathrm{i})}+\frac{\mathrm{FC}_{2}}{(1+\mathrm{i})^{2}}+\frac{\mathrm{FC}_{3}}{(1+\mathrm{i})^{2}}+\ldots+\frac{\mathrm{FC}_{\mathrm{n}}}{(1+\mathrm{i})^{\mathrm{n}}}\right]-\mathrm{FC}_{0}
$$

onde, $\mathrm{FC}_{\mathrm{n}}$ : fluxos de caixa do período, $\mathrm{FC}_{0}$ : investimento inicial, i: custo do capital e Taxa Interna de Retorno [TIR]

Como forma também de analisar financeiramente o projeto corroborando com o VPL, foi utilizada a TIR, que é a taxa interna de retorno do investimento comparada com uma Taxa Mínima de Atratividade [TMA], que será baseada na taxa da Poupança Anual. A TIR é a taxa que anula o VPL, conforme, a eq. (2):

$$
\mathrm{VPL}=-\mathrm{I}+\sum_{\mathrm{t}=1}^{\mathrm{n}} \frac{\mathrm{FC}_{\mathrm{t}}}{\left(1+\mathrm{i}^{*}\right)^{\mathrm{t}}}
$$

onde, I: investimento inicial e $\mathrm{FC}_{\mathrm{t}}$ : fluxo de caixa no tésimo período

Por ser um método eficaz para a análise da variável tempo e capital, foi utilizado o payback para avaliar o tempo de recuperação do investimento. Foram estimados o payback simples e o payback descontado.

O Payback descontado determina o número de períodos necessários para reaver o capital inicialmente aplicado no investimento, considerando o efeito do tempo (juros).

O inventimento inicial foi calculado baseado na eq (3) (Samanez, 2009).

$$
\mathrm{I}=\sum_{\mathrm{t}=1}^{\mathrm{T}} \frac{\mathrm{FC}_{\mathrm{t}}}{(1+\mathrm{K})^{\mathrm{t}}}
$$

onde, I: investimento inicial, FCt: fluxo de caixa no período t e K o custo do capital. O método do Payback descontado consiste, basicamente em determinar o $T$.

\section{Resultados e Discussão}

\section{Retirada dos materiais do campo}

Após a última colheita, retirou-se os restos vegetais da área usando máquinas enfardadeiras, facilitando a remoção do mulching com o trator agrícola e garras laterais nas quais desprendem o material plástico da terra. Após a remoção, o plástico é conduzido pelos carroções para pesagem e posteriormente para a área de seleção.

Procedimento semelhante ocorre com a Manta/TNT, no qual foram conduzidas para a área de seleção e limpeza. Aquelas que foram utilizadas mais de duas vezes ou que estavam com furos ficam inviáveis para utilização em campo.

\section{Análise econômica}

A empresa geradora dos materiais recolheu os plásticos do campo, aferiu o peso e comercializou para a empresa recicladora, cobrando $\mathrm{R} \$ 0,10 \mathrm{~kg}^{-1}$ de material, sendo ele manta ou mulching.

A capacidade de processamento da usina de reciclagem é 90 mil toneladas por mês, sendo menor que o volume de resíduos gerados, o que garante produção ininterrupta durante todo o ano. $\mathrm{O}$ material processado foi comercializado pela empresa recicladora pelo valor de $\mathrm{R} \$ 2,00 \mathrm{~kg}^{-1}$. Os compradores recolhem o material processado na usina e, por meio de processos de beneficiamento, produzem cadeiras plásticas, bacias plásticas, mangueiras e demais artigos plásticos.

Os custos de investimento, incluindo a estrutura e os equipamentos, totalizaram $\mathrm{R} \$ 860.700,00$. Os custos e despesas mensais giraram em torno de $\mathrm{R} \$ 55.000,00$, que somando-se aos impostos cobrados sobre a venda dos materiais, tiveram um custo total de $\mathrm{R} \$ 86.560,00$. Com a venda de $60.000 \mathrm{~kg}$ de material, a receita foi de $\mathrm{R} \$ 120.000,00$.

Os custos e despesas para a reciclagem do material plástico (manta) foram estimados em 77,5\% do montante gerado com sua comercialização, desta forma o lucro líquido girou em torno de 22,5\% (Tabela 1). 
Tabela 1. Custos e despesas da reciclagem de material plástico, manta, utilizado no manejo de culturas frutíferas

\begin{tabular}{|c|c|c|c|c|}
\hline Investimento (Estrutura e equipamentos) & Quantidade & Unidade & Preço & Total \\
\hline & & & \multicolumn{2}{|c|}{ 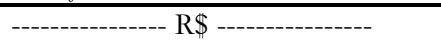 } \\
\hline Escritório/Galpão/Estrutura de contenção & 1 & & $250.000,00$ & $250.000,00$ \\
\hline Caminhão com Garra & 1 & & $370.000,00$ & $370.000,00$ \\
\hline Maquinário - sistema de limpeza do TNT & 1 & & $110.000,00$ & $110.000,00$ \\
\hline Rasgadores & 1 & & $114.000,00$ & $114.000,00$ \\
\hline Guilhotina & 1 & & $15.000,00$ & $15.000,00$ \\
\hline Prensa & 1 & & $1.700,00$ & $1.700,00$ \\
\hline \multirow[t]{2}{*}{ Total } & & & & $860.700,00$ \\
\hline & \multicolumn{4}{|c|}{ Custos e despesas (mensal) } \\
\hline Material agrícola manta & 90.000 & $\mathrm{~kg}$ & 0,10 & $9.000,00$ \\
\hline Frete de retirada & 90.000 & $\mathrm{~kg}$ & 0,10 & $9.000,00$ \\
\hline Energia elétrica (maquinário) & & & & $10.000,00$ \\
\hline Manutenção de equipamentos & & & & $8.000,00$ \\
\hline Outros custos e despesas (embalagens, pigmentos, escritório, etc.) & & & & $5.000,00$ \\
\hline Colaboradores & 7 & & $2.000,00$ & $14.000,00$ \\
\hline Total & & & & $55.000,00$ \\
\hline \multicolumn{5}{|l|}{ Receita (mensal) } \\
\hline Material manta aglutinado & 60000 & $\mathrm{~kg}$ & 2,00 & $120.000,00$ \\
\hline \multicolumn{5}{|l|}{ Impostos } \\
\hline Impostos sobre a venda & 26,3 & $\%$ & & $31.560,00$ \\
\hline Custos totais (mensal) & 72,13 & $\%$ & & $86.560,00$ \\
\hline Receita Líquida (mensal) & 27,87 & $\%$ & & $33.440,00$ \\
\hline Receita Líquida (Anual) & & & & $401.280,00$ \\
\hline
\end{tabular}

Devido a perdas e sujeiras, após processar e limpar 90 toneladas de manta, gerou-se apenas 60 toneladas de material plástico para ser tratado. Confrontando os dados de custos de produção com os dados do lucro líquido gerado pela venda do material, notou-se que há viabilidade na produção deste tipo de atividade, mesmo com os altos encargos tributários exigidos pelo governo e com os elevados custos de energia elétrica e dos materiais de insumo (Tabela 2).

Tabela 2. Fluxo de caixa e indicadores financeiros, Valor Presente Líquido [VPL], Taxa Interna de retorno [TIR], payback e Taxa Mínima de Atratividade [TMA]

\begin{tabular}{|c|c|c|c|c|c|}
\hline Ano & 0 & 1 & 2 & 3 & 4 \\
\hline Fluxo de Caiva Final & -86070000 & 40128000 & 40128000 & ------------- & ----------- \\
\hline $\begin{array}{l}\text { Fluxo de Calxa Final } \\
\text { Fluxo de Caixa Acumulado }\end{array}$ & $-860.700,00$ & $-459.420,00$ & $-58.140,00$ & $343.140,00$ & $744.420,00$ \\
\hline Fluxo de Caixa Descontado & $-860.700,00$ & $378.566,04$ & $357.137,77$ & $336.922,43$ & $317.851,35$ \\
\hline Fluxo de Caixa Descontado Acumulado & $-860.700,00$ & $-482.133,96$ & $-124.996,19$ & $211.926,24$ & $529.777,58$ \\
\hline VPL & $529.777,58$ & & & & \\
\hline TIR & $31,00 \%$ & & & & \\
\hline Payback Simples & Ano: 2 & Mês: 1 & & & \\
\hline Payback Descontado & Ano: 2 & Mês: 4 & & & \\
\hline TMA anual (Base Poupança) & $6,00 \%$ & & & & \\
\hline
\end{tabular}

De acordo com os resultados da análise de viabilidade econômica do investimento, o VPL foi positivo, o que sugere que o retorno do projeto paga o investimento inicial de $\mathrm{R} \$ 860.700,00$. Os cálculos apontaram que pode obter-se o valor de $\mathrm{R} \$ 529.777,58$ a mais ao ano, do que se ganharia com esse montante financeiro investido e aplicado na poupança. A TIR foi de $31 \%$, ou seja, cinco vezes maior que a taxa mínima de atratividade usada nesse trabalho, que teve como base a poupança, sugerindo também que o projeto é viável.
Os cálculos para payback simples mostrou que o tempo de recuperação do investimento foi de dois anos e um mês, para payback descontado dois anos e quatro meses. Para maior segurança na avaliação do projeto, o payback descontado foi mais indicado por corrigir o dinheiro no decorrer do tempo.

Assim como nesse trabalho, Hopewell et al. (2009) também evidenciaram a importância da reciclagem de plásticos utilizados na agricultura, principalmente quanto a classificação eficiente dos materiais de entrada a fim de 
garantir que os diversos tipos de plásticos sejam obtidos com níveis elevados de pureza.

\section{Conclusão}

Em tempo de difícil economia e sérios problemas ambientais, a reciclagem e reutilização de materiais e bens de consumo é de grande importância. As empresas agrícolas também estão se adequando a tal situação e empregando a seu favor tecnologias das quais possam cortar custos e despesas de produção e se manterem competitivos no mercado agrícola.

É viável economicamente trabalhar com reciclagem, especificamente na fruticultura, podendo ser uma segunda fonte de geração de renda de forma ambientalmente correta.

A empresa recicladora deve continuar investindo em novas técnicas e maquinários a fim de tornar o produto final mais refinado e pronto para uso nas indústrias, como também possibilitar a reciclagem de outros materiais usados na produção.

\section{Referências}

Akcelrud, L. 2007. Fundamentos da Ciência dos Polímeros. Manole Editora, São Paulo, SP, Brasil. Canevarolo Jr., S.V. 2004. Ciência dos polímeros: Um Texto Básico para Tecnólogos e Engenheiros. Artliber Editora, São Paulo, SP, Brasil.

Espí, E.; Salmerón, A.; Fontecha, A.; García, Y.; Real, A.I. 2016. Plastic Films for Agricultural Applications. Journal of Plastic Film \& Sheeting 22(2): 85-102.

Gu, F.; Guo, J.; Zhang, W.; Summers, P.A.; Hall, P. 2017. From waste plastics to industrial raw materials: A life cycle assessment of mechanical plastic recycling practice based on a real-world case study. Science of The Total Environment 601: 1191-1207.

Hopewell, J.; Dvorak, R.; Kosior, E. 2009. Plastics recycling: challenges and opportunities. Philosophical Transactions of the Royal Society B: Biological Sciences 364: 2115-2126. doi:10.1098/rstb.2008.0311.

Kyrikou, I.; Briassoulis, D. 2007. Biodegradation of Agricultural Plastic Films: A Critical Review. Journal of Polymers and the Environment 15(2):125-150.

Lamont Jr.; W.J. 2005. Plastics: Modifying the Microclimate for the Production of Vegetable Crops. Hort'Technology 15(3): 477-481.

Neves, J.M. 1994. Perspectivas para o uso de fibras secundárias no Brasil. O Papel 55(2): 40-46.

Pinto, J.M.; Costa, N.D.; Yuri, J.E.; Calgaro, M.; Resende, G.M. 2012. Cultivo de meloeiro irrigado por gotejamento usando mulching de plástico. In: XXII Congresso Nacional de Irrigação e Drenagem, 2012, Cascavel, Paraná. Anais do XXII Congresso Nacional de Irrigação e Drenagem. Pdf número 5037. Disponível em: <http://www.abid.org.br/congressos/download.asp $>$. Acesso em: 31 ago. 2017.

Piva, A.M; Neto, M.B; Wiebeck, H. 1999. A Reciclagem do PVC no Brasil. Polímeros: Ciência e Tecnologia 9(4): 195-200.

Rujnić-Sokele M.; Pilipović A. 2017. Challenges and opportunities of biodegradable plastics: A mini review. Waste Management \& Research 35(2):132140. doi: $10.1177 / 0734242 X 16683272$.

Samanez, C.P. 2002. Matemática financeira: aplicações à análise de Investimentos. Lapponi, Rio de Janeiro, RJ, Brasil.

Samanez, C.P. 2009. Engenharia Econômica. 1ed. Pearson Prentice Hall, São Paulo, SP, Brasil.

Spinacé, M.S.; De Paoli, M.A. 2005. A tecnologia da reciclagem de polímeros. Revista Química Nova 28: 65-72.

Thompson, R.C.; Swan, S.H.; Moore, C.J.; Vom Saal, F.S. 2009. Our plastic age. Philosophical Transactions of the Royal Society B: Biological Sciences 364: 19731976. doi:10.1098/rstb.2009.0054. 